\title{
Article \\ Disparities in ART Live Birth and Cumulative Live Birth Outcomes for Hispanic and Asian Women Compared to White Non-Hispanic Women
}

\author{
Alexander M. Kotlyar ${ }^{1, *}$, Burcin Simsek ${ }^{2}$ and David B. Seifer ${ }^{1}$ \\ 1 Department of Obstetrics, Gynecology and Reproductive Sciences, Yale School of Medicine, \\ New Haven, CT 06510, USA; david.seifer@yale.edu \\ 2 Department of Statistics, University of Pittsburgh, Pittsburgh, PA 15260, USA; burcinsim@gmail.com \\ * Correspondence: alexander.kotlyar@yale.edu
}

Citation: Kotlyar, A.M.; Simsek, B.; Seifer, D.B. Disparities in ART Live Birth and Cumulative Live Birth Outcomes for Hispanic and Asian Women Compared to White Non-Hispanic Women. J. Clin. Med. 2021, 10, 2615. https://doi.org/ $10.3390 / \mathrm{jcm} 10122615$

Academic Editor: Eyal Sheiner

Received: 23 April 2021

Accepted: 10 June 2021

Published: 14 June 2021

Publisher's Note: MDPI stays neutral with regard to jurisdictional claims in published maps and institutional affiliations.

Copyright: (C) 2021 by the authors. Licensee MDPI, Basel, Switzerland. This article is an open access article distributed under the terms and conditions of the Creative Commons Attribution (CC BY) license (https:// creativecommons.org/licenses/by/ $4.0 /)$.

\begin{abstract}
BACKGROUND: Conflicting disparities have been seen in assisted reproductive technology (ART) outcomes for Hispanic and Asian women compared to white, non-Hispanic (WNH) women. We, therefore, sought to clarify these disparities and calculated cumulative live birth rates (CLBR) for these racial or ethnic groups using the SARTCORS database. METHODS: We performed an analysis of the 2014-2016 SARTCORS database for member clinics doing at least 50 cycles of ART each year. RESULTS: In comparison to cycles in WNH women, cycles in Hispanic and Asian patients were in older $(p<0.001)$, more nulliparous women, that were less likely to have a history of endometriosis compared WNH women regardless of prior ART status. ART cycles in Hispanic and Asian women, exhibited lower rates of live birth (LB) per cycle start $(p<0.001)$ compared to cycles in WNH women. Multivariate logistic regression demonstrated that cycles from Hispanic and Asian women were less likely to have a LB and CLBR than white women (OR $0.86 ; p=0.004$, OR $0.69 ; p<0.001$, respectively) independent of age, parity, BMI, etiology of infertility, use of ICSI or number of embryos transferred. CONCLUSIONS: Race or ethnicity continues to be an independent prognostic factor for LB and CLBR for ART. Additional analysis of trends among Hispanic and Asian women is warranted to enable addressing disparities in outcomes in ART treatment.
\end{abstract}

Keywords: racial or ethnic disparities; Hispanic; Asian; non-Hispanic white; live birth rate; cumulative live birth rate; mandated states; non-mandated states; outcomes research

\section{Introduction}

Racial and ethnic disparities in infertility and infertility treatment outcomes are well established [1]. Numerous studies have observed disparities in outcomes between black non-Hispanic (BNH) women and white non-Hispanic $(\mathrm{WNH})$ women in regards to their success using assisted reproductive technologies (ART) for autologous, fresh, non-donor cycles. These disparities include lower clinical intra-uterine gestation (CIG), live birth rate (LBR) per transfer, and LBR per cycle start. Three large database studies have compared outcomes between cycles from $\mathrm{BNH}$ and $\mathrm{WNH}$ women utilizing the Society for Assisted Reproductive Technologies Clinical Outcome Reporting System (SARTCORS) and have demonstrated race as a robust independent predictor of live birth outcomes in ART cycles [1-3].

Although clear disparities have been consistently seen between BNH women and WNH women, the degree of disparity is much less clear for Hispanic and Asian women. A single-center study from 2011 comparing 134 Hispanic patients to $301 \mathrm{WNH}$ patients showed no difference in CIG and SAB rates nor was there any difference noted in LBR. However, they did note that Hispanic women were more likely to have tubal factor infertility and endometriosis [2]. These findings are in contrast to two large scale studies utilizing the SARTCORS database showing lower LBR and higher spontaneous abortion 
(SAB) rates [3,4]. Additional studies from outside the US also showed a lower LBR in Asian women than white-Caucasian women undergoing fresh embryo transfer [5]. Several other single-center studies did not find a difference in CIG rate and LBR, even after controlling for confounding variables such as age, BMI, day $3 \mathrm{FSH}$, smoking status, and infertility diagnosis [6-8]. Therefore, ongoing reporting discrepancies exists concerning ART outcomes in Hispanic and Asian women.

A similar inconsistent situation is noted with women identifying themselves as Asian. Several earlier SARTCORS database studies showed lower clinical pregnancy rates and LBR in Asian compared to WNH women [3,4,9]. However, the most contemporary of these studies examined a cohort over 10 years ago from 2004-2006. In addition, several single-center studies report conflicting results with some showing a lower LBR in Asian women compared to $\mathrm{WNH}$ and others showing no difference $[7,10,11]$.

Nearly 10 years have passed since the question of outcome disparities has been addressed between Hispanic or Asian women and their WNH counterparts. Furthermore, no study has yet to determine if there are disparities in cumulative live birth rate (CLBR) as prior studies have focused only upon the initial cycle that women underwent followed by their immediate fresh transfer [12]. In addition, the question of whether state-mandated insurance coverage has influenced disparities among Hispanic and Asian patients has yet to be examined. We, therefore, sought to address these questions by performing an analysis of ART cycle outcomes in Asian and Hispanic women using the 2014-2016 SARTCORS database.

\section{Materials and Methods}

\subsection{Data Source and Inclusion Criteria}

This was a retrospective cohort study utilizing the 2014-2016 SARTCORS dataset. This study was deemed exempt from review by the institutional review board of the Yale School of Medicine as the dataset was anonymous and de-identified. De-identified data from member clinics comprising over 91\% of reported ART cycles from 2014-2016 from the SARTCORS database were extracted. This data had been validated by SART and were also reported to the Centers for Disease Control as part of the Fertility Clinic Success Rate and Certification Act of 1992. Data fields were validated with 10 of 11 sampled data fields showing discrepancy rates of $5 \%$ or less. The data submitted from member clinics included information about ART treatment cycles and the outcomes of both fresh and frozen autologous cycles, donor cycles, and non-donor embryo transfer cycles based upon a standardized protocol. Race was designated as white non-Hispanic (WNH), Hispanic, Asian, Black non-Hispanic (BNH), Native American or other which is inconsistent with Centers for Disease Control (CDC) definitions of racial categories [13].

The initial SARTCORS dataset was obtained from the SARTCORS data vendor, RedShift Technologies. This initial dataset contained 563,730 ART cycles during the 2014-2016 time period. In total, 1753 cycles were excluded since they were from clinics performing less than 50 cycles per year to avoid sampling bias. A total of 219,171 (39\%) of cycles were excluded from the resultant 561,977 cycles due to missing data on race or ethnicity. Of note, a comparison was done between the 219,171 excluded cycles and the original 561,977 cycles, and the LBR was same in both confirming that this exclusion did not skew the data. In total, 162,632 of the 561,977 original cycles were fresh autologous cycles. A total of 11,530 fresh cycles (6.5\%) were subsequently excluded since donor oocytes were used. Another 2530 fresh autologous cycles $(0.02 \%)$ were excluded since race or ethnicity were reported in multiple categories to minimize confounding. In total, 11,710 non-WNH, non-Hispanic, non-Asian cycles were also excluded. Therefore, 148,572 fresh autologous cycles were included in the study (See Figure 1). Our analysis also assessed cumulative live birth rates (CLBR) which were obtained by linking all embryo transfers to their original index oocyte retrieval cycles for cycles in 2014 and 2015. Our CLBR analysis included 12,885 cycles which had been reported for Hispanic women, 26,683 cycles which had been reported for Asian women, and 109,004 cycles for NHW women. A subgroup analysis cycles was 
performed in states with an insurance mandate which was defined as states mandating third party-payer coverage of ART for the study period of 2014-2016. These mandated states included Arkansas, Connecticut, Hawaii, Illinois, Maryland, Massachusetts, Rhode Island, and New Jersey.

\section{3,730 ART cycles initially reviewed}

\begin{abstract}
219,171 removed
due to missing race data
\end{abstract}

11,530 donor cycles excluded
1753 cycles removed from clinics with less than 50 cycles per year

342,806 cycles remaining

168,644 frozen

autologous cycles excluded

162,632 cycles remaining

14,060 Non-WNH, Non-Hispanic, NonAsian, mixed race cycles excluded
148,572 fresh autologous cycles included

Figure 1. Scheme for SARTCORS database cycle inclusion.

\subsection{Statistical Analysis}

We analyzed the data using R 3.5.1 package for Windows (Microsoft, Redmond, WA, USA). Our unit of analysis was the treatment cycle given that the data were deidentified. Data from cycles in which the women had no prior ART cycle were analyzed separately since these patients may have a more favorable prognosis given lack of prior failed ART cycle. Diagnoses were assessed separately, i.e., if a patient had more than one etiology of infertility, each diagnosis was analyzed individually. Unusually high (outliers) FSH dosage values (e.g., >80 ampules) were removed since they may have been coding errors. Implantation rate was determined by obtaining the quotient of the number of fetal heartbeats in a given cycle and the number of embryos transferred in the cycle. We defined clinical pregnancy rates as the presence of a gestational sac seen via firsttrimester ultrasound. Live birth was defined as the birth of one or more living infants. Rates of each of these parameters were determined per cycle start.

The definition of a cumulative live birth rate (CLBR) was set as the birth of at least one living infant from an associated primary transfer (fresh or frozen and thaw (FET)). SART defines CLBR as the number of embryo transfers associated or linked with a source index retrieval cycle within one year of that retrieval cycle. One limitation of the database is that it does not include start dates for cycles or dates of retrievals and transfers. However, "reporting year" (the year for cycle start) for each cycle is listed in the database. Therefore, we calculated the cumulative rate within a 24-month maximum timeframe. Additional details on how the CLBR was calculated can be found in Seifer et al. [14].

Only two-tailed statistical tests were used with a $p$-value of $<0.05$ being considered significant. Percentages did not equal to 100 due to rounding, and there were different numbers of cycles in some analyses because of missing data. Chi-squared testing was used for categorical variables. Student's $t$-test was used for continuous variables; however, if the distributions were non-normal, a Mann-Whitney test was used. The $95 \%$ confidence intervals were reported for all values. To determine the contribution of race or ethnicity on ART treatment outcomes, multivariable logistic regression analyses were performed by 
adjusting for potential confounding factors including age, parity, BMI, etiology of infertility, use of ICSI, and number of embryos transferred.

\section{Results}

In this study, we analyzed the disparities between Hispanic and Asian women compared to WNH women. Our analysis included 8341 cycles from Hispanic women with no prior ART, and 4544 cycles with Hispanic patients that did undergo prior ART. In total, 14,696 cycles in Asian women with no prior ART and 11,987 cycles in women that did undergo prior ART were analyzed. The Hispanic and Asian cycles with no prior ART were compared to 64,878 cycles in WNH women with no prior ART. For the Asian and Hispanic cycles with prior ART, they were compared to 44,126 cycles from WNH women with prior ART (Tables 1 and 2).

We note clear disparities in age and BMI. For both Hispanic and Asian women, cycles from both women involved significantly older women compared to cycles involving $\mathrm{WNH}$ women $(p<0.001)$. This difference was sustained regardless of whether the cycles were in women with or without prior ART. Asian women tended to have a lower BMI and were more likely to be nulliparous whether they had or had not had prior ART compared to WNH women $(p<0.001)$. Examining cycles from Hispanic women with no prior ART showed greater nulliparity compared to WNH women $(p<0.001)$. (Tables 1 and 2$)$. The opposite BMI trend was seen in Hispanic women $(p<0.001)$.

Concerning etiology of infertility, cycles from Hispanic and Asian women showed substantial divergence. Although cycles from Asian women with tubal factor was not significantly different compared to WNH women regardless of ART history, tubal factor was more prevalent among cycles from Hispanic compared to WNH women in populations with and without prior ART (Tables 1 and 2). Cycles from Asian and Hispanic women were also noted to have a higher prevalence of uterine factor $(p<0.001)$ and diminished ovarian reserve (DOR) $(p<0.001)$, but a lower risk of male factor $(p<0.001)$ and endometriosis $(p<0.001)$ compared to WNH women. Notably, no difference was seen in the proportion of patients with unexplained infertility among Asian women and WNH women. However, unexplained infertility was more prevalent among cycles from $\mathrm{WNH}$ women compared to Hispanic women $(p<0.001)$. Overall, while cycles from Hispanic women were noted to have the greatest prevalence of tubal factor among the three racial or ethnic groups, both Hispanic and Asian cycles were characterized by greater uterine factor and DOR as their underlying infertility etiologies compared to $\mathrm{WNH}$ women. 
Table 1. Baseline characteristics, treatment, and outcomes for fresh, nondonor cycles among Hispanic, Asian, and white women with no prior AT.

\begin{tabular}{|c|c|c|c|c|c|c|c|c|c|c|c|}
\hline \multirow[b]{3}{*}{$\begin{array}{l}\text { Characteristics } \\
\text { (\% Reporting) }\end{array}$} & \multicolumn{5}{|c|}{ No Prior ART } & \multirow[b]{3}{*}{ Characteristics (\% Reporting) } & \multicolumn{5}{|c|}{ No Prior ART } \\
\hline & \multicolumn{2}{|c|}{ Hispanic $(n=8341)$} & \multicolumn{2}{|c|}{ White $(n=64,878)$} & \multirow[b]{2}{*}{$p$} & & \multicolumn{2}{|c|}{ Asian $(n=14,696)$} & \multicolumn{2}{|c|}{ White $(n=64,878)$} & \multirow[b]{2}{*}{$p$} \\
\hline & $\%$ & $95 \% \mathrm{CI}$ & $\%$ & $95 \% \mathrm{CI}$ & & & $\%$ & $95 \% \mathrm{CI}$ & $\%$ & $95 \% \mathrm{CI}$ & \\
\hline Women's age (year) & & & & & $<0.001$ & Women's age (year) & & & & & $<0.001$ \\
\hline$<35$ & 43.4 & $(42.3-44.5)$ & 54.6 & $(54.2-55)$ & & $<35$ & 42.7 & $(41.9-43.5)$ & 54.6 & $(54.2-55)$ & \\
\hline $35-37$ & 22.6 & $(21.7-23.5)$ & 21.2 & $(20.8-21.5)$ & & $35-37$ & 22.6 & $(21.9-23.3)$ & 21.2 & $(20.8-21.5)$ & \\
\hline $38-40$ & 20.1 & $(19.2-20.9)$ & 15.2 & $(14.9-15.4)$ & & $38-40$ & 19 & $(18.4-19.7)$ & 15.2 & $(14.9-15.4)$ & \\
\hline$>42$ & 5.5 & $(5.0-6.0)$ & 3.5 & $(3.3-3.6)$ & & $>42$ & 7 & $(6.6-7.4)$ & 3.5 & $(3.3-3.6)$ & \\
\hline Nulliparous (49.6) & 43.3 & $(41.8-44.8)$ & 51.2 & $(50.5-51.8)$ & $<0.001$ & Nulliparous (48.6) & 55.4 & $(54.1-56.8)$ & 51.2 & $(50.5-51.8)$ & $<0.001$ \\
\hline $\begin{array}{l}\text { Past spontaneous } \\
\text { abortions }\end{array}$ & 24 & $(23.1-24.9)$ & 20.2 & $(19.9-20.5)$ & $<0.001$ & Past spontaneous abortions & 19 & $(18.4-19.7)$ & 20.2 & $(19.9-20.5)$ & 0.001 \\
\hline Diagnosis & & & & & & Diagnosis & & & & & \\
\hline Tubal Factor & 25.6 & $(24.7-26.6)$ & 11 & $(10.8-11.2)$ & $<0.001$ & Tubal Factor & 10.5 & $(10.0-11.0)$ & 11 & $(10.8-11.2)$ & 0.076 \\
\hline Tubal Ligation & 8 & $(7.4-8.6)$ & 1.7 & $(1.6-1.8)$ & $<0.001$ & Tubal Ligation & 0.6 & $(0.5-0.7)$ & 1.7 & $(1.6-1.8)$ & $<0.001$ \\
\hline Hydrosalpinx & 1.5 & $(1.3-1.8)$ & 0.8 & $(0.7-0.8)$ & $<0.001$ & Hydrosalpinx & 1.1 & $(0.9-1.3)$ & 0.8 & $(0.7-0.8)$ & $<0.001$ \\
\hline Other & 16.4 & $(15.6-17.2)$ & 8.6 & $(8.4-8.9)$ & $<0.001$ & Other & 9 & $(8.5-9.4)$ & 8.6 & $(8.4-8.9)$ & 0.192 \\
\hline $\begin{array}{l}\text { History of } \\
\text { endometriosis }\end{array}$ & 7.4 & $(6.8-8.0)$ & 9.2 & $(9-9.4)$ & $<0.001$ & History of endometriosis & 6.9 & $(6.5-7.3)$ & 9.2 & $(9-9.4)$ & $<0.001$ \\
\hline $\begin{array}{c}\text { Diminished ovarian } \\
\text { reserve }\end{array}$ & 26.1 & $(25.2-27.1)$ & 21.5 & $(21.2-21.8)$ & $<0.001$ & Diminished ovarian reserve & 28.5 & $(27.7-29.2)$ & 21.5 & $(21.2-21.8)$ & $<0.001$ \\
\hline Unexplained & 8.3 & $(7.7-8.9)$ & 13.3 & $(13-13.6)$ & $<0.001$ & Unexplained & 13.3 & $(12.8-13.9)$ & 13.3 & $(13-13.6)$ & 0.963 \\
\hline $\begin{array}{l}\text { Ovulation Disorder } \\
\text { (PCOS) }\end{array}$ & 12.3 & $(11.6-13.0)$ & 13.9 & $(13.7-14.2)$ & $<0.001$ & Ovulation Disorder (PCOS) & 11.6 & $(11.1-12.2)$ & 13.9 & $(13.7-14.2)$ & $<0.001$ \\
\hline Other & 19.6 & $(18.7-20.4)$ & 25.5 & $(25.1-25.8)$ & $<0.001$ & Other & 31.4 & $(30.7-32.2)$ & 25.5 & $(25.1-25.8)$ & $<0.001$ \\
\hline Day 3 FSH <10 IU /L & 3.7 & $(3.4-4.2)$ & 4 & $(3.8-4.1)$ & 0.293 & Day 3 FSH <10 IU /L & 3.7 & $(3.5-4.1)$ & 4 & $(3.8-4.1)$ & 0.172 \\
\hline Day $3 \mathrm{FSH}>10 \mathrm{IU} / \mathrm{L}$ & 69 & $(68.0-70.0)$ & 65.4 & $(65.1-65.8)$ & $<0.001$ & Day 3 FSH > $10 \mathrm{IU} / \mathrm{L}$ & 67.8 & $(67.0-68.5)$ & 65.4 & $(65.1-65.8)$ & $<0.001$ \\
\hline $\begin{array}{l}\text { FSH dosage } \geq 37 \\
\text { ampules }(93.7)\end{array}$ & 54.1 & $(52.9-55.2)$ & 52.2 & $(51.8-52.6)$ & 0.002 & $\begin{array}{c}\text { FSH dosage } \geq 37 \text { ampules } \\
\text { (93.5) }\end{array}$ & 53.4 & $(52.6-54.3)$ & 52.2 & $(51.8-52.6)$ & 0.007 \\
\hline $\begin{array}{l}\text { AMH }<1 \text { among } \\
\text { women }<40 \mathrm{yr}\end{array}$ & 13.6 & $(12.8-14.4)$ & 13.4 & $(13.1-13.6)$ & 0.616 & $\mathrm{AMH}<1$ among women $<40 \mathrm{yr}$ & 11.8 & $(11.2-12.4)$ & 13.4 & $(13.1-13.6)$ & $<0.001$ \\
\hline High ovarian response & 0.3 & $(0.2-0.5)$ & 0.5 & $(0.4-0.5)$ & 0.213 & High ovarian response & 0.4 & $(0.3-0.6)$ & 0.5 & $(0.4-0.5)$ & 0.761 \\
\hline
\end{tabular}


Table 1. Cont.

\begin{tabular}{|c|c|c|c|c|c|c|c|c|c|c|c|}
\hline \multirow[b]{3}{*}{$\begin{array}{l}\text { Characteristics } \\
\text { (\% Reporting) }\end{array}$} & \multicolumn{5}{|c|}{ No Prior ART } & \multirow[b]{3}{*}{ Characteristics (\% Reporting) } & \multicolumn{5}{|c|}{ No Prior ART } \\
\hline & \multicolumn{2}{|c|}{ Hispanic $(n=8341)$} & \multicolumn{2}{|c|}{ White $(n=64,878)$} & \multirow[b]{2}{*}{$p$} & & \multicolumn{2}{|c|}{ Asian $(n=14,696)$} & \multicolumn{2}{|c|}{ White $(n=64,878)$} & \multirow[b]{2}{*}{$p$} \\
\hline & $\%$ & $95 \% \mathrm{CI}$ & $\%$ & $95 \% \mathrm{CI}$ & & & $\%$ & $95 \% \mathrm{CI}$ & $\%$ & $95 \% \mathrm{CI}$ & \\
\hline Cycle Cancelled & 10.9 & $(10.2-11.6)$ & 9.9 & $(9.6-10.1)$ & 0.004 & Cycle Cancelled & 10.6 & $(10.1-11.1)$ & 9.9 & $(9.6-10.1)$ & 0.01 \\
\hline Due to low response & 82.4 & $(80.0-84.8)$ & 84.1 & $(83.2-85.0)$ & 0.188 & Due to low response & 84.5 & $(82.6-86.3)$ & 84.1 & $(83.2-85.0)$ & 0.735 \\
\hline ICSI & 62.8 & $(61.8-63.9)$ & 61.2 & $(60.8-61.6)$ & 0.005 & ICSI & 57 & $(56.2-58.7)$ & 61.2 & $(60.8-61.6)$ & $<0.001$ \\
\hline No of embryos & & & & & $<0.001$ & No of embryos & & & & & $<0.001$ \\
\hline 1 & 27.8 & $(26.6-29.0)$ & 38.2 & $(37.7-38.6)$ & & 1 & 42 & $(40.9-43.1)$ & 38.2 & $(37.7-38.6)$ & \\
\hline 2 & 59.4 & $(58.1-60.7)$ & 54.1 & $(53.6-54.6)$ & & 2 & 47 & $(45.9-48.1)$ & 54.1 & $(53.6-54.6)$ & \\
\hline $3+$ & 12.8 & $(11.8-13.8)$ & 7.7 & $(7.5-8)$ & & $3+$ & 11 & $(10.3-11.7)$ & 7.7 & $(7.5-8)$ & \\
\hline $\begin{array}{l}\text { No. of embryos } \\
\text { transferred: mean (sd) }\end{array}$ & \multicolumn{2}{|c|}{$1.88(0.71)$} & \multicolumn{2}{|c|}{$1.72(0.67)$} & $<0.001$ & $\begin{array}{c}\text { No. of embryos transferred: } \\
\text { mean }(\mathrm{sd})\end{array}$ & \multicolumn{2}{|c|}{$1.73(0.79)$} & \multicolumn{2}{|c|}{$1.72(0.67)$} & 0.153 \\
\hline $\begin{array}{l}\text { Implantation rate \%: } \\
\text { mean }(\mathrm{sd})\end{array}$ & \multicolumn{2}{|c|}{$69.71(34.16)$} & \multicolumn{2}{|c|}{$76.52(33.4)$} & $<0.001$ & Implantation rate \%: mean (sd) & \multicolumn{2}{|c|}{$74.26(35.67)$} & \multicolumn{2}{|c|}{$76.52(33.4)$} & 0.001 \\
\hline Treatment outcome & & & & & & Treatment outcome & & & & & \\
\hline $\begin{array}{l}\text { Clinical intrauterine } \\
\text { gestation (CIG) }\end{array}$ & 30.2 & $(29.2-31.2)$ & 33 & $(32.7-33.4)$ & $<0.001$ & $\begin{array}{c}\text { Clinical intrauterine gestation } \\
\qquad(\mathrm{CIG})\end{array}$ & 22.5 & $(22.5-33.0)$ & 33 & $(32.7-33.4)$ & $<0.001$ \\
\hline $\begin{array}{l}\text { Biochemical } \\
\text { pregnancy }\end{array}$ & 5.4 & $(4.9-5.9)$ & 5.7 & $(5.5-5.9)$ & 0.333 & Biochemical pregnancy & 4.6 & $(4.3-5.0)$ & 5.7 & $(5.5-5.9)$ & $<0.001$ \\
\hline Ectopic or heterotopic & 0.6 & $(0.5-0.8)$ & 0.5 & $(0.5-0.6)$ & 0.382 & Ectopic or heterotopic & 0.5 & $(0.4-0.7)$ & 0.5 & $(0.5-0.6)$ & 1 \\
\hline Not preganant & 54.2 & $(53.1-55.3)$ & 47.2 & $(46.8-47.6)$ & $<0.001$ & Not preganant & 51.7 & $(50.9-52.5)$ & 47.2 & $(46.8-47.6)$ & $<0.001$ \\
\hline $\begin{array}{l}\text { Live birth per cycle } \\
\text { started }\end{array}$ & 24.3 & $(23.4-25.3)$ & 27.9 & $(27.6-28.3)$ & $<0.001$ & Live birth per cycle started & 18.2 & $(17.6-18.9)$ & 27.9 & $(27.6-28.3)$ & $<0.001$ \\
\hline Plurality of birth (24.6) & & & & & 0.005 & Plurality of birth (23.1) & & & & & $<0.001$ \\
\hline Singleton & 74.2 & $(72.2-76.0)$ & 76.9 & $(76.2-77.5)$ & & Singleton & 82.1 & $(80.6-83.5)$ & 76.9 & $(76.2-77.5)$ & \\
\hline Twins & 23.9 & $(22.1-25.9)$ & 21.9 & $(21.3-22.5)$ & & Twins & 16.7 & $(15.3-18.1)$ & 21.9 & $(21.3-22.5)$ & \\
\hline Triples or more & 1.9 & $(1.4-2.6)$ & 1.3 & $(1.1-1.5)$ & & Triples or more & 1.2 & $(0.8-1.7)$ & 1.3 & $(1.1-1.5)$ & \\
\hline $\begin{array}{c}\text { eSET }(\%)<38 \text { y } / \text { o } \\
(44.7)\end{array}$ & 16.1 & $(15.2-17.1)$ & 23.9 & $(23.5-24.2)$ & $<0.001$ & $\operatorname{eSET}(\%)<38$ y $/$ o $(42.8)$ & 23.2 & $(22.4-24.1)$ & 23.9 & $(23.5-24.2)$ & 0.178 \\
\hline $\begin{array}{l}\mathrm{BMI} \geq 30 \\
\text { States }\end{array}$ & 38.3 & $(37.3-39.4)$ & 31.5 & $(31.1-31.9)$ & $\begin{array}{l}<0.001 \\
<0.001\end{array}$ & $\begin{array}{l}\text { BMI } \geq 30 \\
\quad \text { States }\end{array}$ & 7.3 & $(6.9-7.7)$ & 31.5 & $(31.1-31.9)$ & $\begin{array}{c}<0.001 \\
0.749\end{array}$ \\
\hline Mandated & 23.9 & $(23.0-24.8)$ & 27.7 & $(27.4-28.1)$ & & Mandated & 28 & $(27.3-28.7)$ & 27.7 & 0 & \\
\hline Non-mandated & 75.9 & $(74.9-76.8)$ & 71.5 & $(71.1-71.8)$ & & Non-mandated & 71.8 & $(71.1-72.5)$ & 71.5 & 0 & \\
\hline
\end{tabular}


Table 2. Baseline characteristics, treatment, and outcomes for fresh, nondonor cycles among Hispanic, Asian, and WNH women with prior ART.

\begin{tabular}{|c|c|c|c|c|c|c|c|c|c|c|c|}
\hline \multirow[b]{3}{*}{$\begin{array}{l}\text { Characteristics } \\
\text { (\% Reporting) }\end{array}$} & \multicolumn{5}{|c|}{ Prior ART } & \multirow[b]{3}{*}{ Characteristics (\% Reporting) } & \multicolumn{5}{|c|}{ Prior ART } \\
\hline & \multicolumn{2}{|c|}{ Hispanic $(n=4544)$} & \multicolumn{2}{|c|}{ White $(n=44,126)$} & \multirow[b]{2}{*}{$p$} & & \multicolumn{2}{|c|}{ Asian $(n=11,987)$} & \multicolumn{2}{|c|}{ White $(n=44,126)$} & \multirow[b]{2}{*}{$p$} \\
\hline & $\%$ & $95 \% \mathrm{CI}$ & $\%$ & $95 \% \mathrm{CI}$ & & & $\%$ & $95 \% \mathrm{CI}$ & $\%$ & $95 \% \mathrm{CI}$ & \\
\hline Women's age (year) & & & & & $<0.001$ & Women's age (year) & & & & & $<0.001$ \\
\hline$<35$ & 23.6 & $(22.4-24.9)$ & 32.7 & $(32.2-33.1)$ & & $<35$ & 21.7 & $(20.9-22.4)$ & 32.7 & $(32.2-33.1)$ & \\
\hline $35-37$ & 22 & $(20.8-23.2)$ & 21.9 & $(21.5-22.3)$ & & $35-37$ & 18.6 & $(17.9-19.3)$ & 21.9 & $(21.5-22.3)$ & \\
\hline $38-40$ & 26.4 & $(25.1-27.7)$ & 22.1 & $(21.7-22.4)$ & & $38-40$ & 24.6 & $(23.8-25.4)$ & 22.1 & $(21.7-22.4)$ & \\
\hline$>42$ & 13 & $(12.0-14.0)$ & 11.7 & $(11.4-12)$ & & $>42$ & 18.7 & $(18.0-19.4)$ & 11.7 & $(11.4-12)$ & \\
\hline Nulliparous (49.6) & 45.7 & $(43.8-47.5)$ & 44.4 & $(43.8-44.9)$ & 0.188 & Nulliparous (48.6) & 50.9 & $(49.7-52.1)$ & 44.4 & $(43.8-44.9)$ & $<0.001$ \\
\hline $\begin{array}{l}\text { Past spontaneous } \\
\text { abortions }\end{array}$ & 34.4 & $(33.1-35.8)$ & 32.8 & $(32.4-33.3)$ & 0.028 & Past spontaneous abortions & 32.3 & $(31.5-33.2)$ & 32.8 & $(32.4-33.3)$ & 0.311 \\
\hline Diagnosis & & & & & & Diagnosis & & & & & \\
\hline Tubal Factor & 22.6 & $(21.4-23.8)$ & 10.4 & $(10.2-10.7)$ & $<0.001$ & Tubal Factor & 10.7 & $(10.1-11.2)$ & 10.4 & $(10.2-10.7)$ & 0.496 \\
\hline Tubal Ligation & 5.3 & $(4.7-6.0)$ & 1 & $(0.9-1.1)$ & $<0.001$ & Tubal Ligation & 0.5 & $(0.3-0.6)$ & 1 & $(0.9-1.1)$ & $<0.001$ \\
\hline Hydrosalpinx & 2 & $(1.6-2.5)$ & 0.7 & $(0.6-0.8)$ & $<0.001$ & Hydrosalpinx & 1.3 & $(1.1-1.6)$ & 0.7 & $(0.6-0.8)$ & $<0.001$ \\
\hline Other & 15.6 & $(14.5-16.7)$ & 8.8 & $(8.6-9.2)$ & $<0.001$ & Other & 9.2 & $(8.7-9.7)$ & 8.8 & $(8.6-9.2)$ & 0.27 \\
\hline $\begin{array}{c}\text { History of } \\
\text { endometriosis }\end{array}$ & 8.8 & $(8.0-9.7)$ & 9.8 & $(9.5-10.1)$ & 0.033 & History of endometriosis & 7.5 & $(7.0-8.0)$ & 9.8 & $(9.5-10.1)$ & $<0.001$ \\
\hline $\begin{array}{l}\text { Diminished ovarian } \\
\text { reserve }\end{array}$ & 44.2 & $(42.8-45.7)$ & 41.3 & $(40.8-41.7)$ & $<0.001$ & Diminished ovarian reserve & 52.4 & $(51.5-53.3)$ & 41.3 & $(40.8-41.7)$ & $<0.001$ \\
\hline Unexplained & 7.2 & $(6.5-8.0)$ & 10.4 & $(10.1-10.7)$ & $<0.001$ & Unexplained & 9.8 & $(9.3-10.3)$ & 10.4 & $(10.1-10.7)$ & 0.059 \\
\hline $\begin{array}{l}\text { Ovulation Disorder } \\
\text { (PCOS) }\end{array}$ & 9.6 & $(8.8-10.5)$ & 10.2 & $(10.0-10.5)$ & 0.183 & Ovulation Disorder (PCOS) & 7.4 & $(7.0-7.9)$ & 10.2 & $(10.0-10.5)$ & $<0.001$ \\
\hline Other & 18.3 & $(17.2-19.5)$ & 21.2 & (20.9-21.6) & $<0.001$ & Other & 23.5 & $(22.7-24.3)$ & 21.2 & $(20.9-21.6)$ & $<0.001$ \\
\hline Day 3 FSH <10 IU /L & 3.2 & $(2.7-3.8)$ & 4 & $(3.8-4.2)$ & 0.014 & Day 3 FSH <10 IU /L & 4 & $(3.7-4.4)$ & 4 & $(3.8-4.2)$ & 0.855 \\
\hline Day 3 FSH >10 IU/L & 77.6 & $(76.4-79.0)$ & 74 & $(73.6-74.4)$ & $<0.001$ & Day 3 FSH >10 IU/L & 76.5 & $(75.8-77.3)$ & 74 & $(73.6-74.4)$ & $<0.001$ \\
\hline $\begin{array}{l}\text { FSH dosage } \geq 37 \\
\text { ampules (93.7) }\end{array}$ & 64.5 & $(63.0-66.0)$ & 64.8 & $(64.3-65.2)$ & 0.716 & $\begin{array}{c}\text { FSH dosage } \geq 37 \text { ampules } \\
(93.5)\end{array}$ & 60.6 & $(59.6-61.5)$ & 64.8 & $(64.3-65.2)$ & $<0.001$ \\
\hline $\begin{array}{c}\text { AMH }<1 \text { among } \\
\text { women }<40 \mathrm{yr}\end{array}$ & 21.6 & $(20.1-23.2)$ & 21 & $(20.6-21.5)$ & 0.471 & $\mathrm{AMH}<1$ among women $<40 \mathrm{yr}$ & 18.6 & $(17.7-19.6)$ & 21 & $(20.6-21.5)$ & $<0.001$ \\
\hline High ovarian response & 1.1 & $(0.9-1.5)$ & 0.7 & $(0.7-0.8)$ & 0.005 & High ovarian response & 1.2 & $(1.1-1.5)$ & 0.7 & $(0.7-0.8)$ & $<0.001$ \\
\hline Cycle Cancelled & 15.3 & $(14.3-16.4)$ & 13.3 & $(13.0-13.6)$ & $<0.001$ & Cycle Cancelled & 13 & $(12.4-13.6)$ & 13.3 & $(13.0-13.6)$ & 0.302 \\
\hline
\end{tabular}


Table 2. Cont.

\begin{tabular}{|c|c|c|c|c|c|c|c|c|c|c|c|}
\hline \multirow[b]{3}{*}{$\begin{array}{l}\text { Characteristics } \\
\text { (\% Reporting) }\end{array}$} & \multicolumn{5}{|c|}{ Prior ART } & \multirow[b]{3}{*}{ Characteristics (\% Reporting) } & \multicolumn{5}{|c|}{ Prior ART } \\
\hline & \multicolumn{2}{|c|}{ Hispanic $(n=4544)$} & \multicolumn{2}{|c|}{ White $(n=44,126)$} & \multirow[b]{2}{*}{$p$} & & \multicolumn{2}{|c|}{ Asian $(n=11,987)$} & \multicolumn{2}{|c|}{ White $(n=44,126)$} & \multirow[b]{2}{*}{$p$} \\
\hline & $\%$ & $95 \%$ CI & $\%$ & $95 \% \mathrm{CI}$ & & & $\%$ & $95 \% \mathrm{CI}$ & $\%$ & $95 \%$ CI & \\
\hline Due to low response & 74.4 & $(70.9-77.6)$ & 80.1 & $(79.1-81.1)$ & 0.001 & Due to low response & 76.1 & $(73.9-78.2)$ & 80.1 & $(79.1-81.1)$ & 0.001 \\
\hline ICSI & 60.9 & $(59.5-62.3)$ & 63.9 & $(63.4-64.3)$ & $<0.001$ & ICSI & 58.5 & $(57.6-59.4)$ & 63.9 & $(63.4-64.3)$ & $<0.001$ \\
\hline No of embryos & & & & & $<0.001$ & No of embryos & & & & & $<0.001$ \\
\hline 1 & 21.8 & $(20.2-23.4)$ & 26.3 & $(25.8-26.8)$ & & 1 & 28.2 & $(27.0-29.4)$ & 26.3 & $(25.8-26.8)$ & \\
\hline $3+$ & 22.5 & $(21.0-24.2)$ & 20.6 & $(20.1-21.1)$ & & $3+$ & 23.5 & $(22.3-24.6)$ & 20.6 & (20.1-21.1) & \\
\hline $\begin{array}{l}\text { No. of embryos } \\
\text { transferred: mean (sd) }\end{array}$ & \multicolumn{2}{|c|}{$2.08(0.86)$} & \multicolumn{2}{|c|}{$2.02(0.88)$} & $<0.001$ & $\begin{array}{c}\text { No. of embryos transferred: } \\
\text { mean (sd) }\end{array}$ & \multicolumn{2}{|c|}{$2.08(1.03)$} & \multicolumn{2}{|c|}{$2.02(0.88)$} & $<0.001$ \\
\hline $\begin{array}{c}\text { Implantation rate \%: } \\
\text { mean }(\mathrm{sd})\end{array}$ & \multicolumn{2}{|c|}{$60.83(33.08)$} & \multicolumn{2}{|c|}{$67.08(34.1)$} & $<0.001$ & Implantation rate \%: mean (sd) & \multicolumn{2}{|c|}{$63.71(36.73)$} & \multicolumn{2}{|c|}{$67.08(34.1)$} & $<0.001$ \\
\hline Treatment outcome & & & & & & Treatment outcome & & & & & \\
\hline $\begin{array}{l}\text { Clinical intrauterine } \\
\text { gestation (CIG) }\end{array}$ & 22.5 & $(21.3-23.8)$ & 25.1 & $(24.7-25.5)$ & $<0.001$ & $\begin{array}{c}\text { Clinical intrauterine gestation } \\
\qquad(\mathrm{CIG})\end{array}$ & 15.6 & $(15.0-16.3)$ & 25.1 & $(24.7-25.5)$ & $<0.001$ \\
\hline Spontaneous abortion & 23.2 & $(20.6-25.9)$ & 18.6 & $(17.8-19.3)$ & $<0.001$ & Spontaneous abortion & 23.8 & $(21.9-25.8)$ & 18.6 & $(17.8-19.3)$ & $<0.001$ \\
\hline $\begin{array}{l}\text { Biochemical } \\
\text { pregnancy }\end{array}$ & 4.9 & $(4.3-5.6)$ & 5.7 & $(5.5-6.0)$ & 0.025 & Biochemical pregnancy & 4.3 & $(3.9-4.6)$ & 5.7 & $(5.5-6.0)$ & $<0.001$ \\
\hline Ectopic or heterotopic & 0.6 & $(0.4-0.9)$ & 0.5 & $(0.4-0.6)$ & 0.533 & Ectopic or heterotopic & 0.4 & $(0.3-0.5)$ & 0.5 & $(0.4-0.6)$ & 0.109 \\
\hline Not preganant & 62.8 & $(61.4-64.2)$ & 58.3 & $(57.8-58.7)$ & $<0.001$ & Not preganant & 63.2 & $(62.3-64.0)$ & 58.3 & $(57.8-58.7)$ & $<0.001$ \\
\hline $\begin{array}{l}\text { Live birth per cycle } \\
\text { started }\end{array}$ & 16.9 & $(15.8-18.0)$ & 20.2 & $(19.8-20.6)$ & $<0.001$ & Live birth per cycle started & 11.7 & $(11.2-12.3)$ & 20.2 & $(19.8-20.6)$ & $<0.001$ \\
\hline Plurality of birth (24.6) & & & & & 0.217 & Plurality of birth (23.1) & & & & & 0.019 \\
\hline Singleton & 76.5 & $(73.4-79.5)$ & 75.8 & $(74.9-76.6)$ & & Singleton & 79.2 & $(77.0-81.3)$ & 75.8 & $(74.9-76.6)$ & \\
\hline Twins & 21.1 & $(18.4-24.2)$ & 22.6 & $(21.8-23.5)$ & & Twins & 19.4 & $(17.4-21.6)$ & 22.6 & $(21.8-23.5)$ & \\
\hline Triples or more & 2.3 & $(1.4-3.7)$ & 1.6 & $(1.3-1.9)$ & & Triples or more & 1.4 & $(0.8-2.2)$ & 1.6 & $(1.3-1.9)$ & \\
\hline $\begin{array}{c}\text { eSET }(\%)<38 \text { y } / o \\
(44.7)\end{array}$ & 10.4 & $(9.2-11.9)$ & 13.9 & $(13.4-14.3)$ & $<0.001$ & $\operatorname{eSET}(\%)<38$ y/o $(42.8)$ & 13.2 & $(12.2-14.2)$ & 13.9 & $(13.4-14.3)$ & 0.22 \\
\hline $\begin{array}{l}\text { BMI } \geq 30 \\
\quad \text { States }\end{array}$ & 39.9 & $(38.5-41.3)$ & 34.5 & $(34.1-35.0)$ & $\begin{array}{c}<0.001 \\
0.001\end{array}$ & $\begin{array}{l}\mathrm{BMI} \geq 30 \\
\quad \text { States }\end{array}$ & 6.4 & $(6.0-6.9)$ & 34.5 & $(34.1-35.0)$ & $\begin{array}{l}<0.001 \\
<0.001\end{array}$ \\
\hline Mandated & 30.1 & $(28.8-31.5)$ & 32.4 & $(32.0-32.9)$ & & Mandated & 27.3 & $(26.5-28.1)$ & 32.4 & $(32.0-32.9)$ & \\
\hline Non-mandated & 69.8 & $(68.4-71.1)$ & 67 & $(66.5-67.4)$ & & Non-mandated & 72.6 & (71.8-73.4) & 67 & $(66.5-67.4)$ & \\
\hline
\end{tabular}


Substantial disparities were also noted in ART cycle outcomes. Cycles from Asian women with no prior ART were noted to have greater rates of cycle cancelation $(p=0.01)$ compared to WNH women; however, this was not seen in women with prior ART. ICSI was utilized for more cycles from Asian women compared to $\mathrm{WNH}$ women regardless of prior ART usage $(p<0.001)$. Interestingly, Asian women tended to have a significantly greater number of cycles in which 1 embryo or 3 or more embryos were produced $(p<0.001)$. Despite this, implantation rates were significantly lower for cycles from Asian women among both the prior and no prior ART groups. In addition, Asian cycles exhibited broadly lower ART success rates with lower clinical intrauterine gestation (CIG) rates, higher spontaneous abortion (SAB) rates, and lower LBR per CIG, as well as LBR per cycle start compared to WNH cycles. No difference was seen in ectopic and heterotopic rates (Tables 1 and 2) between the three groups of women. Aside from higher cycle cancelation rates in women with prior ART, cycles from Hispanic women exhibited similar disparities in outcomes as Asian women compared to WNH women. Of note, Hispanic women had cycles which led to greater production of 2 or more embryos compared to $\mathrm{WNH}$ women $(p<0.001)$. Yet, ART outcomes were not improved despite a slightly higher number of embryos transferred for women with and without prior ART (Tables 1 and 2).

To assess if race or ethnicity had an independent effect on ART outcomes, we performed a multivariable regression analysis for both Hispanic and Asian cycles with and without prior ART. For Hispanic women, race remained an independent predictor of live birth resulting from an ART cycle with a lower chance of live birth for cycles from Hispanic women compared to cycles from WNH women (OR 0.86, 95\% CI 0.77-0.95 for women without prior ART (initial cycle), OR 0.80, 95\% CI 0.70-0.91 for women with prior ART (Table 3)). This finding was independent of age, BMI, cause of infertility, history of past spontaneous abortions, use of ICSI and number of embryos transferred. When examining cycles from Asian women, we noted similar results with Asian ethnicity as being an independent predictor of live birth regardless whether or not the cycle was associated with prior ART (OR 0.68, 95\% CI 0.62-0.75 for women without prior ART (initial index cycle), OR $0.81,95 \%$ CI $0.74-0.88$ for women with prior ART (Table 3)).

We next examined cumulative live birth rates (CLBR) for cycles in Hispanic and Asian women compared to WNH women for those with and without prior ART. For Hispanic women, race remained a predictor of a lower cumulative live birth rate compared to $\mathrm{WNH}$ even when controlling for age, parity, history of spontaneous abortions, cause of infertility, diminished ovarian reserve, Day $3 \mathrm{FSH}, \mathrm{AMH}$, ICSI, and number of embryos transferred (OR 0.84, 95\% CI: 0.77-0.92) (Table 4). A similar situation was observed for cycles from Asian women when controlling for the same confounders showing Hispanic ethnicity was associated with a lower cumulative live birth rate compared to cycles from WNH women (OR 0.79, 95\% CI: 0.71-0.85, $p<0.001$ ) (Table 4). 
Table 3. Independent predictors of achieving live birth among Hispanic, Asian, and WNH women.

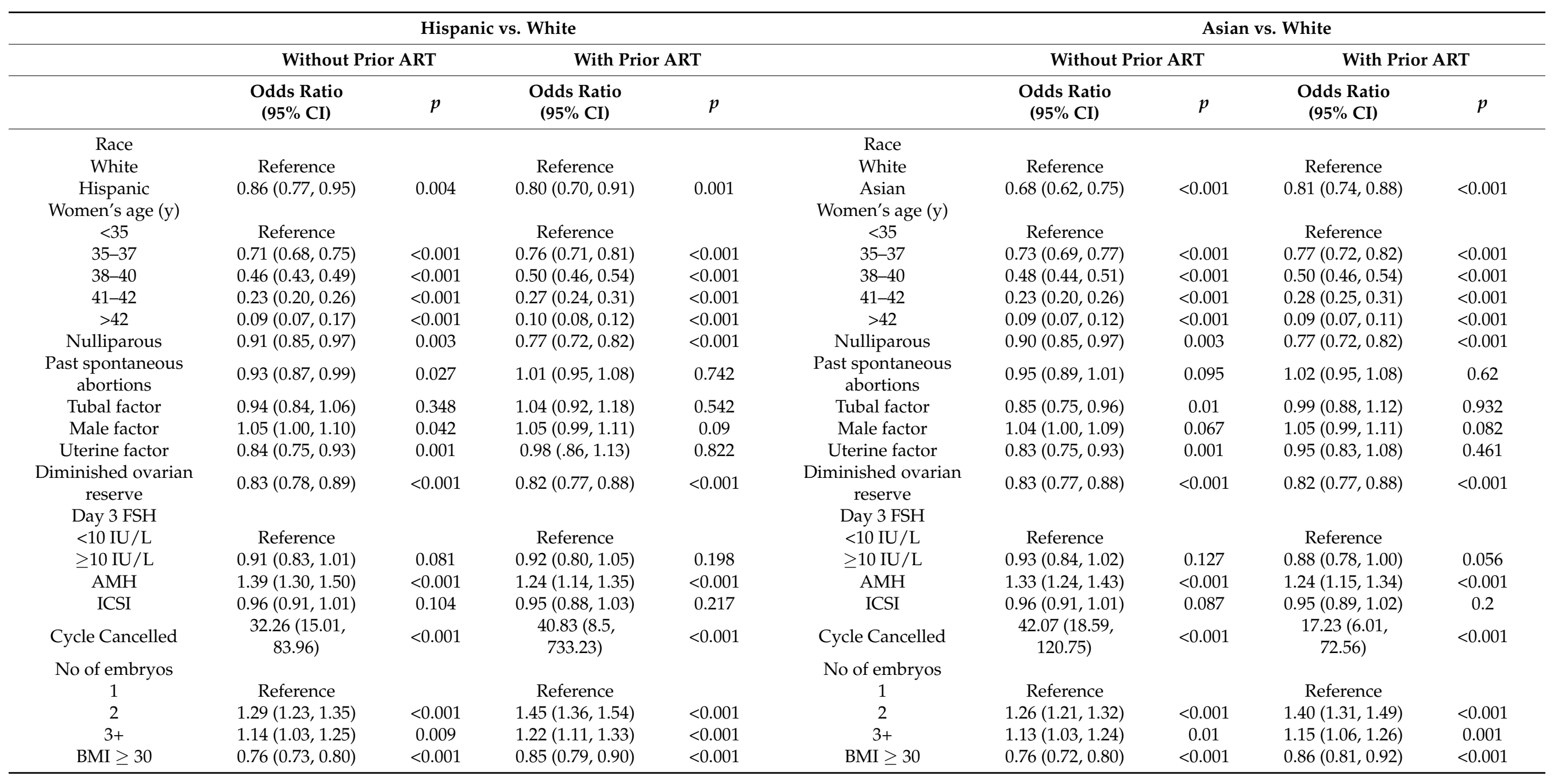


Table 4. Cumulative live birth rate for primary transfer (fresh or thawed FET) in Hispanic, Asian, and WNH women for 2014-2015.

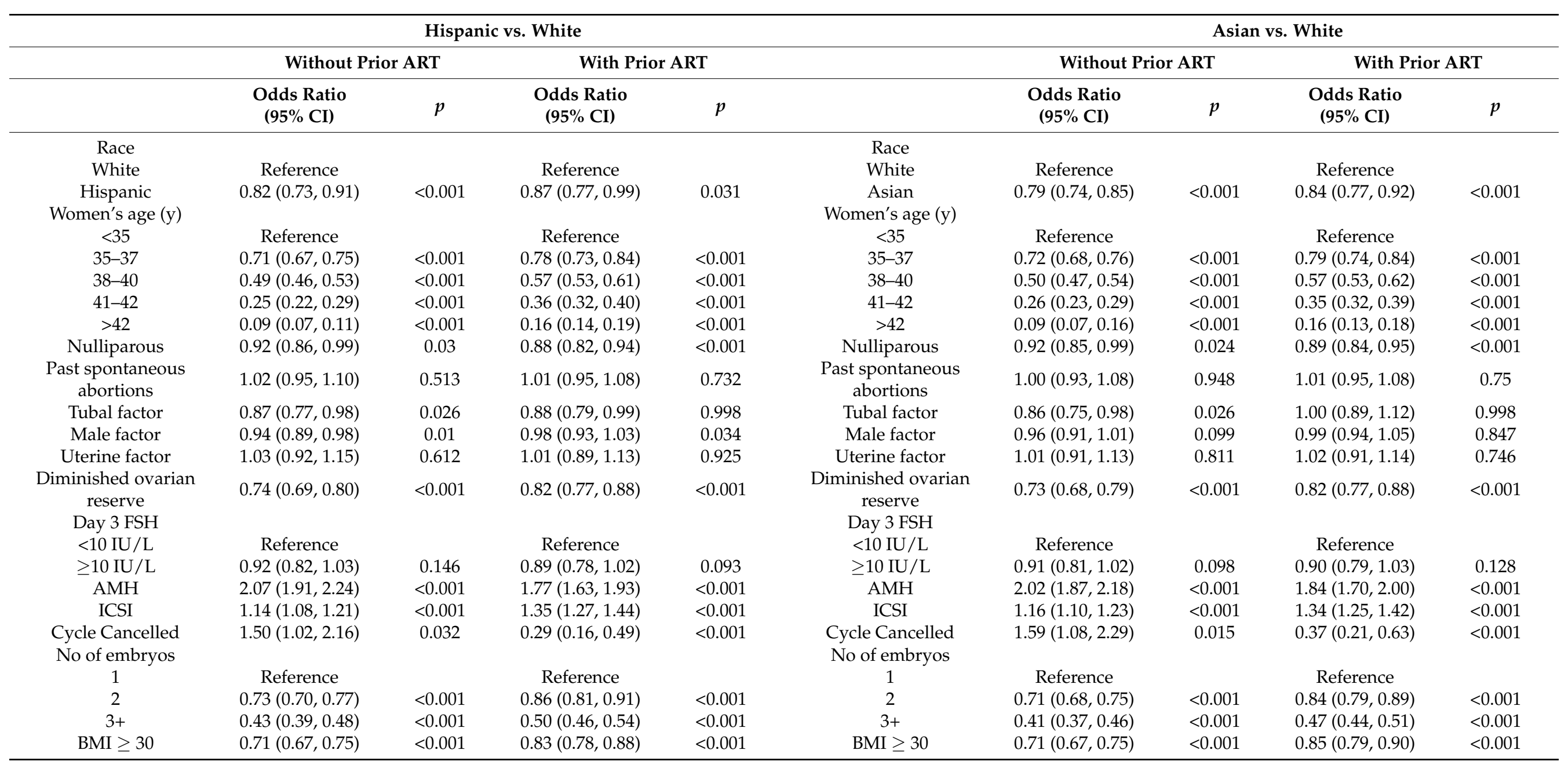




\section{Discussion}

In this study, we assessed the ART outcomes of cycles from Hispanic and Asian women in the 2014-2016 SARTCORS dataset. Compared to cycles from WNH women, cycles from Hispanic women exhibited a greater proportion of tubal factor and a greater frequency of uterine factor and DOR compared to cycles from WNH women. Cycles from both ethnic or racial groups showed lower proportions of CIG rate and LBR compared to cycles from WNH women. In addition, cycles from Hispanic and Asian women exhibited significantly lower CLBR in contrast to WNH women. These differences were independent of age, BMI, cause of infertility, past spontaneous abortions, use of ICSI and number of embryos transferred. This study represents the largest analysis of ART cycle outcomes from Hispanic and Asian women compared to cycles from WNH women to present. In addition, it is the first such study to analyze CLBR between all three groups.

The disparities noted in the data of ART cycle outcomes for Asian and Hispanic women are likely to be multifactorial. This may be secondary to differences in accessto-care and socio-economic status (SES). Both racial or ethnic groups we examined in the current study have a lower SES compared to WNH patients [15]. Lower SES is a well-established factor that is associated with poorer health outcomes [16]. In addition, cycles from both Hispanic and Asian women were overall older and more nulliparous than cycles from WNH women. Although this in-of-itself can lead to a lower LBR given the rapid decline of ovarian reserve with advancing age, $\mathrm{AMH}$ also tends to be lower for Asian and Hispanic women when stratified for age compared to WNH, especially at younger-to-middle ages [17-19]. Furthermore, the combination of older age and greater frequency of nulliparity suggests that Hispanic and Asian women are presenting for ART treatment at a later point in their life cycle for their initial pregnancy attempt as compared to WNH women. The social, cultural, and economic factors influencing these findings of older age and greater nulliparity require future study to determine a better understanding of the underlying causes. Of note, obesity has previously been shown to be associated with poorer ART outcomes in Hispanic patients and obese Asian patients [20]. Although the significantly greater proportion of obesity among Hispanic women in our population may be contributing to the lower LBR of those cycles without prior ART (initial cycles) and prior ART cycles, we observed a significantly lower percentage of obesity among Asian women. This suggests that despite the substantially healthier BMI profile of Asian women, this was not enough to offset the more deleterious influence of older age impacting the lower LBR compared to cycles from WNH women.

These data support several previously defined features of Hispanic and Asian women undergoing ART. Consistent with Fujimoto et al [6] and Shuler et al [4], tubal factor was noted to be greater among Hispanic women compared to WNH women [2,4]. This is consistent with prior work showing greater Chlamydia infection rates among Hispanic women with an adjusted odds ratio (aOR) of 1.46 (95\% CI: 1.33-1.61) compared to WNH women [21]. One notable difference is that our study did find a lower LBR for cycles in Hispanic women compared to WNH women which Shuler et al did not [2]. This difference may be secondary to the smaller sample size resulting in less power to determine a real difference and an increased possibility of a Type 2 error when examining 134 Hispanic and $301 \mathrm{WNH}$ women involved in the latter study.

When examining the clinical characteristics of ART cycles for both Hispanic women and Asian, stark contrasts are noted. In cycles in women without prior ART, the percentage of elective single embryo transfer (eSET) was significantly lower among Hispanic women (16.1\%) compared to WNH women (23.9\%). However, no difference was seen for cycles in Asian women compared to $\mathrm{WNH}$ women without prior ART. This difference confirms data from a prior SARTCORS database analysis in 2010 showing higher percentages of eSET among Asian women compared to WNH and lower eSET rates in Hispanic women [22]. Such consistency indicates that over the subsequent six years the difference in performance of eSET has persisted. One possible explanation for the difference in eSET is the proportion of women with AMH levels less than $1 \mathrm{ng} / \mathrm{mL}$ in women less than 40 years of age which is 
consistent with diminished ovarian reserve. Among all three racial or ethnic groups, this proportion was lowest among cycles in Asian women and the highest among Hispanic women representing an inverse relationship between these variables.

A key novel feature of this study was the analysis of linked cycles for 2014 and 2015 that involved primary transfers of either fresh or frozen and thawed embryos from a single linked retrieval. This is notable in the CLBR among all age groups older than 35 years from cycles from Asian women and Hispanic women compared to WNH women. Throughout all ages, CLBR was lower in cycles in Hispanic and Asian women compared to WNH women. Furthermore, this disparity widened with increasing age thus emphasizing the lower prognostic outlook for non-white women in older age categories.

The presence of state insurance mandates for ART coverage may be one possible explanation for contributing to these disparities. For patients without prior ART in mandated states, the percentage of cycles were $74.6 \%$ for $\mathrm{WNH}$ women, $8.2 \%$ for Hispanic women, and $17.1 \%$ for Asian women. In non-mandated states this distribution was similar with $73.3 \%$ of cycles in WNH women, $10.0 \%$ in Hispanic women, and $16.7 \%$ in Asian women When looking at patients with prior ART cycles in mandated states, the percentage of cycles were $75.5 \%$ for $\mathrm{WNH}$ women, $7.2 \%$ for Hispanic women, and $17.3 \%$ for Asian women. Although for patients with prior ART in non-mandated states this distribution was comparable with $70.5 \%$ of cycles in WNH women, $7.6 \%$ in Hispanic women, and $21.9 \%$ in Asian women. We can place these percentages in context when we consider the racial distribution of mandated states with $69.5 \%$ of women being WNH, $14.1 \%$ being Hispanic, and $9.7 \%$ being Asian. For non-mandated states, the racial distribution is $81.2 \% \mathrm{WNH}, 12.1 \%$ Hispanic, and 3.7\% Asian [23]. Therefore, in mandated states, Asians tended to utilize ART less than expected for their portion of the population and $\mathrm{WNH}$ women and Hispanic women tend to utilize ART more than expected. As for non-mandated states, both Hispanic and Asians used ART more than expected for their portion of the population. Hence the presence of an insurance mandate did not seem to enhance ART use in the Hispanic and Asian populations. This is surprising given the greater use of ART for other racial groups such as black women in mandated states compared to non-mandated states in comparison to NHW [14]. Furthermore, prior work assessing ART access via non-SARTCORS databases has observed greater numbers of ART cycles in Hispanic and Asian women in mandated states [24]. Considering that prior work has shown no difference in infertility rates between races or ethnicities and that insurance access does affect utilization of ART, the presence of an insurance mandate for ART does not seem to be a modifiable factor to improve ART access for Hispanic and Asian women $[25,26]$.

The reasons for the surprising lack of increased ART utilization in mandated states among Hispanic and Asian patients may be multi-fold. First studies of socioeconomic status distribution among various racial and ethnic groups indicate that Hispanic and Asian women have a socioeconomic status that is intermediate between $\mathrm{WNH}$ women and black women [15]. Hence, there could be a threshold SES beyond which a state insurance mandate may not enhance access to ART treatments. Second, even with the presence of an insurance mandate, the structure of coverage for infertility treatment in a state may bias a patient towards more conservative treatments such as ovulation induction with or without intra-uterine insemination depending on the cost-effectiveness assessment by the insurance company within that state [27].

Several strengths and limitations are inherent to the SARTCORS database. First, this database is the largest de-identified, standardized, and validated source of ART data collected from clinics throughout the US. Not only does this enhance the statistical power of this study, it also enhances the generalizability of these findings given the varied ethnic and racial make-up of the United States. Furthermore, this database compiles data from a nationwide network of ART clinics over the span of decades which also allows for identification of and analysis of trends in ART practice and outcomes. Despite the above advantages, a weakness is the lack of information on socioeconomic status of the patients undergoing these ART cycles. Markers of socioeconomic status, such as annual salaries or 
surrogate indicators such as a highest obtained educational level, remain a key confounder when assessing the impact of race or ethnicity on ART outcomes. An additional limitation of the database is the presence of missing data. In total, $39 \%$ of cycles reported in SARTCORS did not have data identifying race or ethnicity even though it is a required field. Race itself a self-reported field which may become more subjective as the population of the US becomes more heterogeneous over time. Furthermore, aside from the general race categories which are consistent with CDC race definitions, there is no further subdivision with each group, i.e., Asians being separated into Chinese, South Asian, South-East Asian, etc. Such subdivisions also exhibit differences in ART outcomes [28].

Despite these acknowledged limitations these data indicate that race or ethnicity remains an independent factor of outcome and access to ART in the US for Hispanic and Asian women. These data further suggest that several factors may have contributed to these disparities and that efforts can be made to mitigate these factors. Such mitigating efforts could involve enhancing education on the age-based decline in fertility to various racial or ethnic groups with consideration to seeking care at a younger age when initially trying to conceive, adequate healthy diet and exercise to maintain a normal BMI, in addition to an expansion of available insurance coverage. Utilizing these efforts may eventually narrow and, ideally, eliminate the ART outcome gaps noted in Hispanic and Asian women.

Author Contributions: A.M.K. was involved in project design, analysis and the composition of the manuscript. B.S. was involved in the analysis. D.B.S. was involved in project design, composition and revision of the manuscript. All authors have read and agreed to the published version of the manuscript.

Funding: No sources of funding were used to conduct this study.

Institutional Revie Board Statement: This study was deemed exempt from review by the Yale University Integrated Research Enterprise Solution Internal Review Board (IRES-IRB) due to use of deidentified data from the SART database.

Informed Consent Statement: This was a retrospective study, no informed consent was required.

Data Availability Statement: The data that support the findings of this study are available from SARTCORS but restrictions apply to the availability of these data, which were used under license for the current study, and so are not publicly available. Data are however available from the authors upon reasonable request and with permission of SARTCORS.

Acknowledgments: SART thanks its members for providing clinical information to the SART Clinic Outcome Reporting System database for use by patients and researchers. Without the efforts of our members, this research would not have been possible.

Conflicts of Interest: The authors do not have any conflicts to disclose.

\section{References}

1. Huddleston, H.G.; Cedars, M.I.; Sohn, S.H.; Giudice, L.C.; Fujimoto, V.Y. Racial and ethnic disparities in reproductive endocrinology and infertility. Am. J. Obstet. Gynecol. 2010, 202, 413-419. [CrossRef]

2. Shuler, A.; Rodgers, A.K.; Budrys, N.M.; Holden, A.; Schenken, R.S.; Brzyski, R.G. In vitro fertilization outcomes in Hispanics versus non-Hispanic whites. Fertil. Steril. 2011, 95, 2735-2737. [CrossRef]

3. Baker, V.L.; Luke, B.; Brown, M.B.; Alvero, R.; Frattarelli, J.L.; Usadi, R.; Grainger, D.A.; Armstrong, A.Y. Multivariate analysis of factors affecting probability of pregnancy and live birth with in vitro fertilization: An analysis of the Society for Assisted Reproductive Technology Clinic Outcomes Reporting System. Fertil. Steril. 2010, 94, 1410-1416. [CrossRef] [PubMed]

4. Fujimoto, V.Y.; Luke, B.; Brown, M.B.; Jain, T.; Armstrong, A.; Grainger, D.A.; Hornstein, M.D.; Society for Assisted Reproductive Technology Writing Group. Racial and ethnic disparities in assisted reproductive technology outcomes in the United States. Fertil. Steril. 2010, 93, 382-390. [CrossRef]

5. Mascarenhas, M.; Balen, A.H. Could ethnicity have a different effect on fresh and frozen embryo transfer outcomes: A retrospective study. Reprod. BioMed. Online 2019, 39, 764-769. [CrossRef] [PubMed]

6. Bendikson, K.; Cramer, D.W.; Vitonis, A.; Hornstein, M.D. Ethnic background and in vitro fertilization outcomes. Int. J. Gynaecol. Obstet. 2005, 88, 342-346. [CrossRef] [PubMed]

7. McQueen, D.B.; Schufreider, A.; Lee, S.M.; Feinberg, E.C.; Uhler, M.L. Racial disparities in in vitro fertilization outcomes. Fertil. Steril. 2015, 104, 398-402. [CrossRef] [PubMed] 
8. $\quad$ Feinberg, E.C.; Larsen, F.W.; Wah, R.M.; Alvero, R.J.; Armstrong, A.Y. Economics may not explain Hispanic underutilization of assisted reproductive technology services. Fertil. Steril. 2007, 88, 1439-1441. [CrossRef] [PubMed]

9. Karen, P.; Michael, S.; Frazier, L.M.; Rall, M.J.; Shen, S.; Croughan, M.; Grainger, D.A.; Fujimoto, V.Y. Asian ethnicity is associated with reduced pregnancy outcomes after assisted reproductive technology. Fertil. Steril. 2007, 87, 297-302.

10. Kan, A.; Leung, P.; Luo, K.; Fay, L.; Tan, C.L. Do Asian women do as well as their Caucasian counterparts in IVF treatment: Cohort study. J. Obstet. Gynaecol. Res. 2015, 41, 946-951. [CrossRef] [PubMed]

11. Langen, E.S.; Shahine, L.K.; Lamb, J.D.; Lathi, R.B.; Milki, A.A.; Fujimoto, V.Y.; Westphal, L.M. Asian ethnicity and poor outcomes after in vitro fertilization blastocyst transfer. Obstet. Gynecol. 2010, 115, 591-596. [CrossRef] [PubMed]

12. Humphries, L.A.; Chang, O.; Humm, K.; Sakkas, D.; Hacker, M.R. Influence of race and ethnicity on in vitro fertilization outcomes: Systematic review. Am. J. Obstet. Gynecol. 2016, 214, 212.e1-212.e17. [CrossRef] [PubMed]

13. Centers for Disease Control: National Health Interview Survey. Available online: Cdc.gov/nchs/nhis/rhoi/rhoi_glossary.htm (accessed on 11 May 2021).

14. Seifer, D.B.; Simsek, B.; Wantman, E.; Kotlyar, A. Status of Racial Disparities Between Black and White Women Undergoing Assisted Reproductive Technology in the US. Reprod. Biol. Endocrinol. 2020, 18, 1-13. [CrossRef] [PubMed]

15. Shariff-Marco, S.; Yang, J.; John, E.M.; Kurian, A.W.; Cheng, I.; Leung, R.; Koo, J.; Monroe, K.R.; Henderson, K.E.; Bernstein, L.; et al. Intersection of Race/Ethnicity and Socioeconomic Status in Mortality After Breast Cancer. J. Community Health 2015, 40, 1287-1299. [CrossRef] [PubMed]

16. Khariton, Y.; Nassif, M.E.; Thomas, L.; Fonarow, G.C.; Mi, X.; DeVore, A.D.; Duffy, C.; Sharma, P.P.; Albert, N.M.; Patterson, J.H.; et al. Health Status Disparities by Sex, Race/Ethnicity, and Socioeconomic Status in Outpatients With Heart Failure. JACC Heart Fail. 2018, 6, 465-473. [CrossRef]

17. Seifer, D.B.; Golub, E.T.; Lambert-Messerlian, G.; Benning, L.; Anastos, K.; Watts, D.H.; Cohen, M.H.; Karim, R.; Young, M.A.; Minkoff, H.; et al. Variations in serum müllerian inhibiting substance between white, black, and Hispanic women. Fertil. Steril. 2009, 92, 1674-1678. [CrossRef] [PubMed]

18. Seifer, D.B.; Baker, V.L.; Leader, B. Age-specific serum anti-Mullerian hormone values for 17.120 women presenting to fertility centers within the United States. Fertil. Steril. 2011, 95, 747-750. [CrossRef] [PubMed]

19. Bleil, M.E.; Gregorich, S.E.; Adler, N.E.; Sternfeld, B.; Rosen, M.P.; Cedars, M.I. Race/ethnic disparities in reproductive age: An examination of ovarian reserve estimates across four race/ethnic groups of healthy, regularly cycling women. Fertil. Steril. 2014, 101, 199-207. [CrossRef]

20. Luke, B.; Brown, M.B.; Stern, J.E.; Missmer, S.A.; Fujimoto, V.Y.; Leach, R. Racial and ethnic disparities in assisted reproductive technology pregnancy and live birth rates within body mass index categories. Fertil. Steril. 2011, 95, 1661-1666. [CrossRef]

21. Fine, D.; Thomas, K.K.; Nakatsukasa-Ono, W.; Marrazzo, J. Chlamydia positivity in women screened in family planning clinics: Racial/ethnic differences and trends in the northwest US 1997-2006. Public Health Rep. 2012, 127, 38-51. [CrossRef]

22. Luke, B.; Brown, M.B.; Grainger, D.A.; Cedars, M.; Klein, N.; Stern, J.E. Practice patterns and outcomes with the use of single embryo transfer in the United States. Fertil. Steril. 2010, 93, 490-498. [CrossRef] [PubMed]

23. United States Census Bureau. QuickFacts United States. Available online: https://www.census.gov/quickfacts/fact/table/US/ PST0452192019 (accessed on 14 April 2021).

24. Dieke, A.C.; Zhang, Y.; Kissin, D.M.; Barfield, W.D.; Boulet, S.L. Disparities in Assisted Reproductive Technology Utilization by Race and Ethnicity, United States, 2014: A Commentary. J. Womens Health 2017, 26, 605-608. [CrossRef] [PubMed]

25. Kelley, A.S.; Qin, Y.; Marsh, E.E.; Dupree, J.M. Disparities in accessing infertility care in the United States: Results from the National Health and Nutrition Examination Survey, 2013-2016. Fertil. Steril. 2019, 112, 562-568. [CrossRef]

26. Quinn, M.; Fujimoto, V. Racial and ethnic disparities in assisted reproductive technology access and outcomes. Fertil. Steril. 2016, 105, 1119-1123. [CrossRef] [PubMed]

27. Hamilton, B.H.; Jungheim, E.; McManus, B.; Pantano, J. Health Care Access, Costs, and Treatment Dynamics: Evidence from In Vitro Fertilization. Am. Econ. Rev. 2018, 108, 3725-3777. [CrossRef]

28. Maalouf, W.; Maalouf, W.; Campbell, B.; Jayaprakasan, K. Effect of Ethnicity on Live Birth Rates after In Vitro Fertilisation/Intracytoplasmic Sperm Injection Treatment: Analysis of UK National Database. BJOG Int. J. Obstet. Gynaecol. 2017, 124, 904-910. [CrossRef] [PubMed] 